\title{
Stability analysis of pulsed cascaded Raman lasers in dispersion-managed systems
}

\author{
Salih K. Kalyoncu, ${ }^{1}$ Shiming Gao, ${ }^{1}$ En-Kuang Tien, ${ }^{1}$ Yuewang Huang, ${ }^{1}$ Dogukan Yildirim, ${ }^{1}$ Enver Adas, \\ Stefan Wabnitz, ${ }^{2}$ and Ozdal Boyraz ${ }^{1,3, *}$ \\ ${ }^{1}$ Electrical Engineering \& Computer Science Department, University of California, Irvine, California 92697, USA \\ ${ }^{2}$ Department of Information Engineering, University of Brescia, 25123 Brescia, Italy \\ ${ }^{3}$ Electrical Engineering Department, founding member, Istanbul Sehir University, Istanbul, Turkey \\ ${ }^{*}$ Corresponding author: oboyraz@uci.edu
}

Received July 15, 2011; revised September 19, 2011; accepted September 19, 2011; posted September 26, 2011 (Doc. ID 151012); published October 31, 2011

\begin{abstract}
We investigate synchronous first and second order pulsed Raman lasers that can achieve frequency spacing of up to $1000 \mathrm{~cm}^{-1}$ for CARS microscopy applications. In particular, we focus on analytical and numerical analysis of pulsed stability derived for Raman lasers by using dispersion-managed telecom fibers and pumping at near $1530 \mathrm{~nm}$ telecom wavelengths. We show the evolution of the first and second order Stokes signals at the output for different peak pump power and the net anomalous dispersion combinations. We determine the stability condition for dispersion-managed synchronous Raman lasers up to second order. The results show that the stable second order Raman Stokes pulses with $0.02 \mathrm{~W}$ to $0.1 \mathrm{~W}$ peak power and $1 \mathrm{ps}$ to $2.1 \mathrm{ps}$ pulse width can be achieved in proposed dispersion-managed systems. (C) 2011 Optical Society of America OCIS codes: $\quad 140.3510,140.3538,140.3550$
\end{abstract}

\section{INTRODUCTION}

Wavelength tunable synchronous pulse sources are highly desirable for spectroscopy and optical diagnostics due to their diverse advantages such as broadband operation, low cost, compactness, and adaptability with fibers [1-3]. The common method to generate short pulses in the fiber is the use of optical nonlinearities and soliton pulse shaping in the anomalous dispersion regime that results in spectral broadening and pulse compression [4]. However, to generate ultrashort pulses, a broadband gain mechanism is also required. Stimulated Raman scattering with a wide gain bandwidth of $\sim 6 \mathrm{THz}$ has an ability to generate femtosecond pulses [5,6]. Additionally, Raman gain can be generated at any wavelength in a nonlinear medium, which facilitates generation of pulses at wavelengths that are not attainable by a conventional laser gain medium. Self-soliton frequency shift (SSFS) of optical pulses in a highly nonlinear fiber (HNLF), such as photonic crystal fiber, due to the self-Raman scattering effect enables tunable femtosecond pulse generation over a range of $>100 \mathrm{~nm}$ [7]. Fixed-wavelength Raman lasers have been widely studied for the past two decades. Recently, much focus has been shifted toward multi-wavelength tunable Raman fiber lasers that can generate output Stokes pulses in a broad wavelength range by the so called cascaded stimulated Raman

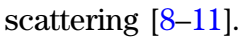

For spectroscopy and optical diagnostics, phase coherent and wavelength tunable synchronous pulse sources are of great demand. In particular, in chemistry and biological applications, higher-order cascaded pulsed Raman lasers with up to $1000 \mathrm{~cm}^{-1}$ frequency spacing is highly desirable for CARS microscopy. Previous experimental works have been dedicated to address that requirement [12-14]. In this paper, we propose and analyze the stability of a new configuration for first and the second order pulsed Raman lasers with a frequency tuning range up to $1000 \mathrm{~cm}^{-1}$. We carry out a detailed numerical analysis in order to investigate the stability regime of the generation of Stokes pulses up to the second order in a dispersion-managed (DM) fiber ring cavity pumped by a $1530 \mathrm{~nm}$ fiber laser. We will show that stable Stokes pulses can be generated with different pump power levels and lengths of the single mode fiber (SMF) that is included in the DM map that forms the cavity. A variational analysis, more powerful and elaborate than the one used in [15] and in our own recent related study [16], is also employed in order to analytically investigate the steady-state dynamics of Stokes pulses inside the cavity and to predict the output pulse parameters by solving ordinary differential equations with a periodic boundary conditions that is a computationally efficient alternative to time consuming full numerical simulations. The analytical results are compared with simulation results to show good agreement with the derived equations. The results illustrate that the stable second order Raman Stokes pulses with $0.02 \mathrm{~mW}$ to $0.1 \mathrm{~W}$ peak power and $1 \mathrm{ps}$ to $2.1 \mathrm{ps}$ pulse width can be achieved in our DM Raman laser system.

\section{SIMULATION SETUP AND VARIATIONAL EQUATIONS}

In order to generate stable pulsed lasers by the Raman process, inventing a proper dispersion management of the pump, first order, and second order pulses is the most important issue. Most of the commercial dispersion shifted fibers and SMFs have high dispersion values and slopes; hence it is not possible to maintain a reasonably limited walk-off (which reduces the time overlapping of pulses) through a significant nonlinear interaction length. In order to effectively eliminate such walk-off, we propose the use of the special DM map inside the ring laser configuration, which is illustrated in Figs. 1 and 2 . The setup of Fig. 1 can be readily experimentally 
implemented: in our work we perform a detailed numerical stability analysis in order to optimize its parameters. Our laser is synchronously pumped by a $1530 \mathrm{~nm}$ mode locked laser with $10 \mathrm{ps}$ pulse width. The laser cavity is designed as proposed in [15], which mainly consists of two cascaded sections, the DM system as the gain mechanism, and the SMF for pulse shaping. The overlapping of Stokes pulses at the beginning is satisfied by inserting a stretcher (delay line) in one of the ring cavities to match the pump repetition rate.

Dispersion management is formed by cascading HNLF pieces, which have a nearly zero dispersion and dispersion slope in a periodic configuration. In particular, we restrict our analysis to commercially available fibers from different vendors. The characteristics of the HNLFs and the SMF used in the simulations are summarized in Table 1 .

In order to raise the net dispersion to anomalous regime, SMF is cascaded with the DM system to generate stable soliton-like pulses. The Stokes pulse evolution in our ring laser configuration of Fig. 1 is modeled by assuming that a single (pump and Stokes) pulse recirculates in the cavity. Therefore, each round trip in the laser involves propagation through a single period of the DM map as in Fig. 2, which illustrates the dispersion profile for each Stokes order. Stokes pulses are generated through the propagation in the ring laser from the noise level, which is taken as the initial condition. When the system reaches a steady state, the Stokes pulses exactly repeat themselves at each period of the map. Thus, the spatial periodic boundary conditions imply that the initial pulse parameters should be the same as the ones at the end of the cavity.

The mutual interaction between the pump pulse and first and second order Stokes pulses is governed by a set of three nonlinear Schrödinger (NLS) equations including all processes of group velocity dispersion (GVD), self-phase modulation (SPM), cross phase modulation (XPM), walk-off between the pulses, Raman gain, and pump depletion [17]:

$$
\begin{aligned}
& \frac{\partial u_{p}}{\partial z}+\frac{1}{\nu_{g p}} \frac{\partial u_{p}}{\partial t}-i \frac{\beta_{2 p}}{2} \frac{\partial^{2} u_{p}}{\partial t^{2}}+i \gamma\left|u_{p}\right|^{2} u_{p} \\
& =-\frac{\alpha}{2} u_{p}-\frac{g_{R}}{2} \frac{\lambda_{1}}{\lambda_{p}}\left|u_{1}\right|^{2} u_{p}-i \gamma\left(2\left|u_{1}\right|^{2}+2\left|u_{2}\right|^{2}\right) u_{p},
\end{aligned}
$$

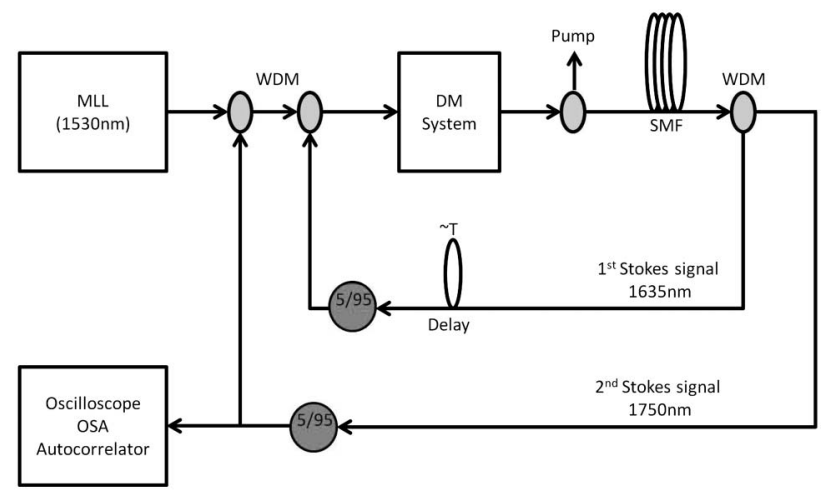

Fig. 1. Designed setup for first and second order Stokes signals.

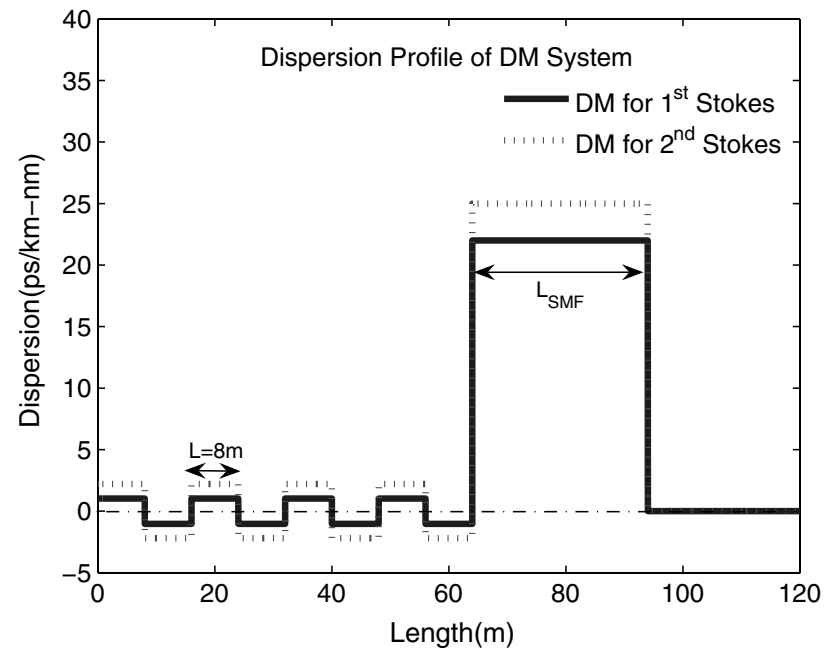

Fig. 2. DM system for each Stokes pulses.

$$
\begin{aligned}
& \frac{\partial u_{1}}{\partial z}+\frac{1}{\nu_{g 1}} \frac{\partial u_{1}}{\partial t}-i \frac{\beta_{21}}{2} \frac{\partial^{2} u_{1}}{\partial t^{2}}+i \gamma\left|u_{1}\right|^{2} u_{1} \\
& =-\frac{\alpha}{2} u_{1}+\frac{g_{R}}{2}\left(\left|u_{p}\right|^{2}-\frac{\lambda_{2}}{\lambda_{1}}\left|u_{2}\right|^{2}\right) u_{1}-i \gamma\left(2\left|u_{p}\right|^{2}+2\left|u_{2}\right|^{2}\right) u_{1},
\end{aligned}
$$

$$
\begin{aligned}
& \frac{\partial u_{2}}{\partial z}+\frac{1}{\nu_{g 2}} \frac{\partial u_{2}}{\partial t}-i \frac{\beta_{22}}{2} \frac{\partial^{2} u_{2}}{\partial t^{2}}+i \gamma\left|u_{2}\right|^{2} u_{2} \\
& \quad=-\frac{\alpha}{2} u_{2}+\frac{g_{R}}{2}\left|u_{1}\right|^{2} u_{2}-i \gamma\left(2\left|u_{p}\right|^{2}+2\left|u_{1}\right|^{2}\right) u_{2},
\end{aligned}
$$

where $\nu_{g p, g 1, g 2}$ is the group velocity, $\beta_{2 p, 21,22}$ is the GVD parameter $\left(\mathrm{ps}^{2} / \mathrm{km}\right), \alpha$ is the fiber loss $\left(\mathrm{km}^{-1}\right), g_{R}$ is the Raman gain coefficient $\left(\mathrm{W}^{-1} \mathrm{~km}^{-1}\right), \gamma$ is the nonlinearity coefficient $\left(\mathrm{W}^{-1} \mathrm{~km}^{-1}\right.$ ), and $u_{p, 1,2}$ is the amplitude of the pump, first order Stokes, and the second order Stokes signals, respectively. These governing equations include all the possible interactions among three optical waves. Four-wave mixing, which cannot build up coherently due to the short length of the HNLFs and the high net dispersion of the cavity, as well as the self-Raman induced frequency shift, which is negligible for pulses with $>1$ ps pulse width, are ignored in this analysis. However, time-consuming numerical analysis is required to determine the pulse evolution in the laser cavity and in optical fibers by using these equations.

Table 1. Characteristics of the Fibers Used in the Simulations [20]

\begin{tabular}{lccc}
\hline & $\begin{array}{c}\text { HNLF (+) } \\
\text { Slope }\end{array}$ & $\begin{array}{c}\text { HNLF (-) } \\
\text { Slope }\end{array}$ & SMF \\
\hline$\alpha$ : Fiber loss $(\mathrm{dB} / \mathrm{km})$ & 0.9 & 0.9 & 0.2 \\
$\gamma:$ Nonlinearity coefficient & 11.5 & 11.5 & 2 \\
$\quad(1 / \mathrm{W}-\mathrm{km})$ & 11.7 & 11.7 & 50 \\
$A_{\text {eff }}\left(\mu \mathrm{m}^{2}\right)$ & 3.4 & 3.4 & 0.8 \\
$g_{R}:$ Raman coefficient & & & \\
$(1 / \mathrm{W}-\mathrm{km})$ & $1530 / 0.01$ & $1530 /-0.01$ & $1310 / .076$ \\
$\lambda_{0}:$ Zero dispersion $(\mathrm{nm}) / \mathrm{slope}$ \\
$\quad\left(\mathrm{ps} / \mathrm{nm}^{2} \mathrm{~km}\right)$
\end{tabular}


Analytical modeling may substantially alleviate computational requirements. Here, we developed an analytical model for the propagation of pump and the Stokes pulses. The pump pulse, which is repetitive at each round trip, is used up in the system and cannot preserve its input Gaussian envelope $\left(\left|u_{p}(0, t)\right|^{2}=P_{p 0} \exp \left[-\frac{\left(t-t_{p}\right)^{2}}{T_{p}^{2}}\right]\right.$, where $P_{p 0}$ is the peak power, $T_{p}$ is the pulse width, and $t_{p}$ is the temporal position). The NLS equation for the pump pulse [Eq. (1)] can be simplified by neglecting dispersive effects (the GVD parameter at the pump wavelength is zero and the dispersion length is much longer than the cavity length) and by separating spectral (for phase accumulation) and temporal terms (for the pulse shape) so as to solve pump propagation analytically. Fiber loss and pump depletion (as seen in Eq. (ㅁ), which provides the pump temporal evolution) are the dominant terms that affect the pulse shape as follows:

$$
\frac{\partial u_{p}}{\partial z} \approx-\frac{\alpha}{2} u_{p}-\frac{g_{R}}{2} \frac{\lambda_{1}}{\lambda_{p}}\left|u_{1}\right|^{2} u_{p}
$$

The pump signal is depleted by the first Stokes signal through Raman interaction. Since the walk-off between the pump and the first Stokes signal is very small, saturation in the gain line shows up locally by causing hole burning at the center of the pulse. The evolution of the pump pulse is approximated by solving Eq. ()ㅜㅇ as

$$
\begin{gathered}
\left|u_{p}(z, t)\right|^{2}=P_{p 0} \exp \left[-\frac{\left(t-t_{p}\right)^{2}}{T_{p}^{2}}\right] \\
\times \exp \left[-\int_{0}^{z}\left(\alpha+g_{R} \frac{\lambda_{1}}{\lambda_{p}}\left|u_{1}\right|^{2}\right) \mathrm{d} z^{\prime}\right] \\
\left|u_{p}(z, t)\right|^{2} \approx P_{p 0} \exp \left[-\frac{\left(t-t_{p}\right)^{2}}{T_{p}^{2}}\right]\left[1-\int_{0}^{z}\left(\alpha+g_{R} \frac{\lambda_{1}}{\lambda_{p}}\left|u_{1}\right|^{2}\right) \mathrm{d} z^{\prime}\right] \\
=\left|u_{p}(0, t)\right|^{2}-\left|u_{h}(z, t)\right|^{2} .
\end{gathered}
$$

The second term $\left[u_{h}(z, t)\right]$ in Eq. () represents hole burning at the center of the pulse. The hole is modeled by a Gaussian envelope with the peak power $\left(P_{h}\right)$, the pulse width $\left(T_{h}\right)$, and the temporal position $\left(t_{h}\right)$. All loss mechanisms such as fiber loss and gain depletion lead to the growth of the hole (peak power). Since the walk-off between the pump and the first Stokes pulses $(0.42 \mathrm{ps})$ is much smaller than the pulse width, the pump and the hole are assumed to be temporally cocentered $\left(t_{h} \cong t_{p}\right)$. In addition, due to relatively small change in the pulse width of the pump and the first Stokes pulses in the DM section at steady state, the pulse width of the hole is approximated as a combination of pump and first Stokes pulse widths. The hole burning is approximated as

$$
\begin{aligned}
\frac{d P_{h}}{d z} & \approx \alpha P_{p 0}+g_{R} \frac{\lambda_{1}}{\lambda_{p}} P_{1} P_{p 0} \text { and } T_{h}(z) \\
& \approx\left(\frac{1}{T_{p}^{2}}+\frac{1}{T_{1}^{2}}\right)^{-1 / 2} \text { and } t_{h} \approx t_{p} .
\end{aligned}
$$

The propagation of Stokes pulses is modeled by means of the variational analysis method, which assumes the signal maintains its shape even though its power, pulse width, and chirp changes in a continuous manner and remains the same periodically after each round trip. The evolution of single pulse parameters in the variational method [18] for the unperturbed NLS equations is evaluated by using the Lagrangian density:

$$
L=\frac{i}{2}\left(u \frac{\partial u^{*}}{\partial z}-u^{*} \frac{\partial u}{\partial z}\right)+\frac{\beta_{2}}{2}\left|\frac{\partial u}{\partial t}\right|^{2}+\frac{\gamma}{2}\left|u^{4}\right|
$$

The unperturbed NLS equation is derived from a Lagrangian that is defined as the integration of Lagrangian density with respect to time:

$$
L=\int_{-\infty}^{\infty} L\left(u, u_{z}, u_{t} ; u^{*}, u_{z}^{*}, u_{t}^{*}\right) \mathrm{d} t
$$

where the subscripts $z$ and $t$ correspond to differentiation with respect to distance and time, respectively. The evolution of the pulse parameters is derived by employing modified Euler-Lagrange equations, taking into account signal to signal interactions such as Raman amplification, XPM, and walk-off as a perturbation to the system. In particular, the physical effects of XPM and temporal walk-off are dominant for determining the chirp evolution, the pump depletion, and the gain reduction [19]. The Euler-Lagrange equation reads as

$$
\frac{\partial L}{\partial x}-\frac{d}{d z}\left(\frac{\partial L}{\partial x_{z}}\right)=\operatorname{Im}\left\{\int_{-\infty}^{\infty}\left[R \frac{\partial u^{*}}{\partial x}-R^{*} \frac{\partial u}{\partial x}\right] \mathrm{d} t\right\},
$$

where $x$ corresponds to any one of the six pulse parameters, such as the energy, the pulse width, the temporal position, the chirp, the central frequency, and the phase, Im denotes the imaginary part, and $R$ includes all the perturbation terms analyzed in the right side of the coupled NLS equations. The Gaussian ansatz is assumed for both first and second order Stokes pulses as the pulse envelope [18]:

$$
u=\sqrt{\frac{E}{T}} \frac{1}{\sqrt[4]{\pi}} \exp \left[-\left(\frac{\left(t-t_{0}\right)^{2}}{2 T^{2}}\right)(1+i C)+i \Omega\left(t-t_{0}\right)+i \varphi\right]
$$

where $E$ is the energy, $T$ is the pulse width, $t_{0}$ is the temporal position, $C$ is the chirp parameter, $\Omega$ is the center frequency, and $\varphi$ is the phase of the pulse. According to the proposed ansatz, the Lagrangian is defined as

$$
\begin{aligned}
L= & E\left[-\frac{1}{4} \frac{d C}{d z}+\frac{C}{2 T} \frac{d T}{d z}+\frac{\gamma E}{2 \sqrt{2 \pi} T}+\frac{\beta_{2}\left(1+C^{2}\right)}{4 T^{2}}\right. \\
& \left.+\frac{d \phi}{d z}+t_{0} \frac{d \Omega}{d z}+\frac{\beta_{2} \Omega^{2}}{2}\right] .
\end{aligned}
$$

By substituting the pulse envelope [Eq. (7)] and the Lagrangian [Eq. (ㅇ)] with a Gaussian ansatz into the perturbed Euler-Lagrange equation [Eq. (ㅁ)], we derived the modified variational equations for the Stokes pulse parameters, pulse energy $(E)$, pulse width $(T)$, chirp $(C)$, temporal position $\left(t_{0}\right)$, and center frequency $(\Omega)$ as 


$$
\begin{aligned}
\frac{d E_{1}}{d z}= & -\frac{\alpha E_{1}}{T_{1} \sqrt{\pi}} I_{0,1,0}\left(0, t_{1}, 0\right) \\
& +\frac{g_{R} E_{1}}{T_{1} \sqrt{\pi}}\left(J_{0,1,1}\left(0, t_{1}, t_{p}\right)-\frac{\lambda_{2}}{\lambda_{1}} \frac{E_{2}}{T_{2} \sqrt{\pi}} I_{0,1,1}\left(0, t_{1}, t_{2}\right)\right),
\end{aligned}
$$

$$
\begin{aligned}
\frac{d T_{1}}{d z}= & \frac{2 T_{1}}{E_{1}}\left[-\frac{1}{4} \frac{d E_{1}}{d z}-\frac{E_{1} \beta_{21} C_{1}}{2 T_{1}^{2}}-\frac{\alpha E_{1}}{2 T_{1}^{3} \sqrt{\pi}} I_{2,1,0}\left(t_{1}, t_{1}, 0\right)\right. \\
& \left.+\frac{g_{R}}{2 T_{1}^{3}} \frac{E_{1}}{\sqrt{\pi}}\left(J_{2,1,1}\left(t_{1}, t_{1}, t_{p}\right)-\frac{\lambda_{2}}{\lambda_{1}} \frac{E_{2}}{T_{2} \sqrt{\pi}} I_{2,1,1}\left(t_{1}, t_{1}, t_{2}\right)\right)\right],
\end{aligned}
$$

$$
\begin{aligned}
\frac{d C_{1}}{d z}= & -\frac{2 T_{1}}{E_{1}}\left[\frac{C_{1}}{2 T_{1}} \frac{d E_{1}}{d z}+\frac{E_{1} \beta_{21}\left(1+C_{1}^{2}\right)}{2 T_{1}^{3}}+\frac{\gamma E_{1}^{2}}{T_{1}^{2} 2 \sqrt{2 \pi}}\right. \\
& +\frac{\alpha E_{1} C_{1}}{T_{1}^{4} \sqrt{\pi}} I_{2,1,0}\left(t_{1}, t_{1}, 0\right) \\
& -\frac{g_{R} E_{1} C_{1}}{T_{1}^{4} \sqrt{\pi}}\left(J_{2,1,1}\left(t_{1}, t_{1}, t_{p}\right)-\frac{\lambda_{2}}{\lambda_{1}} \frac{E_{2}}{T_{2} \sqrt{\pi}} I_{2,1,1}\left(t_{1}, t_{1}, t_{2}\right)\right) \\
& +\frac{2 \gamma E_{1}}{T_{1}^{2} \sqrt{\pi}}\left(J_{0,1,1}\left(0, t_{1}, t_{p}\right)+\frac{E_{2}}{T_{2} \sqrt{\pi}} I_{0,1,1}\left(0, t_{1}, t_{2}\right)\right) \\
& \left.-\frac{4 \gamma E_{1}}{T_{1}^{4} \sqrt{\pi}}\left(J_{2,1,1}\left(t_{1}, t_{1}, t_{p}\right)+\frac{E_{2}}{T_{2} \sqrt{\pi}} I_{2,1,1}\left(t_{1}, t_{1}, t_{2}\right)\right)\right],
\end{aligned}
$$

$$
\begin{aligned}
\frac{d t_{1}}{d z}= & w_{12}-\frac{1}{E_{1}}\left[\frac{d E_{1}}{d z} t_{1}-E_{1} \beta_{21} \Omega_{1}+\frac{\alpha E_{1}}{T_{1} \sqrt{\pi}} I_{1,1,0}\left(t_{1}, t_{1}, 0\right)\right. \\
& \left.-\frac{g_{R} E_{1}}{T_{1} \sqrt{\pi}}\left(J_{1,1,1}\left(t_{1}, t_{1}, t_{p}\right)-\frac{\lambda_{2}}{\lambda_{1}} \frac{E_{2}}{T_{2} \sqrt{\pi}} I_{1,1,1}\left(t_{1}, t_{1}, t_{2}\right)\right)\right]
\end{aligned}
$$

$$
\begin{aligned}
\frac{d \Omega_{1}}{d z}= & \frac{1}{E_{1}}\left[\frac{\alpha E_{1} C_{1}}{T_{1}^{3} \sqrt{\pi}} I_{1,1,0}\left(t_{1}, t_{1}, 0\right)\right. \\
& -\frac{g_{R} E_{1} C_{1}}{T_{1}^{3} \sqrt{\pi}}\left(J_{1,1,1}\left(t_{1}, t_{1}, t_{p}\right)-\frac{\lambda_{2}}{\lambda_{1}} \frac{E_{2}}{T_{2} \sqrt{\pi}} I_{1,1,1}\left(t_{1}, t_{1}, t_{2}\right)\right) \\
& -\frac{\alpha E_{1} \Omega_{1}}{T_{1} \sqrt{\pi}} I_{0,1,0}\left(0, t_{1}, 0\right) \\
& +\frac{g_{R} E_{1} \Omega_{1}}{T_{1}^{3} \sqrt{\pi}}\left(J_{0,1,1}\left(t_{1}, t_{1}, t_{p}\right)-\frac{\lambda_{2}}{\lambda_{1}} \frac{E_{2}}{T_{2} \sqrt{\pi}} I_{0,1,1}\left(t_{1}, t_{1}, t_{2}\right)\right) \\
& \left.-\frac{4 \gamma E_{1}}{T_{1}^{3} \sqrt{\pi}}\left(J_{1,1,1}\left(t_{1}, t_{1}, t_{p}\right)+\frac{E_{2}}{T_{2} \sqrt{\pi}} I_{1,1,1}\left(t_{1}, t_{1}, t_{2}\right)\right)\right],
\end{aligned}
$$

$$
\frac{d E_{2}}{d z}=-\frac{\alpha E_{2}}{T_{2} \sqrt{\pi}} I_{0,1,0}\left(0, t_{2}, 0\right)+\frac{g_{R} E_{2}}{T_{2} \sqrt{\pi}} \frac{E_{1}}{T_{1} \sqrt{\pi}} I_{0,1,1}\left(0, t_{2}, t_{1}\right)
$$

$$
\begin{aligned}
\frac{d T_{2}}{d z}= & \frac{2 T_{2}}{E_{2}}\left[-\frac{1}{4} \frac{d E_{2}}{d z}-\frac{E_{2} \beta_{22} C_{2}}{2 T_{2}^{2}}-\frac{\alpha E_{2}}{2 T_{2}^{3} \sqrt{\pi}} I_{2,1,0}\left(t_{2}, t_{2}, 0\right)\right. \\
& \left.+\frac{g_{R}}{2 T_{2}^{3}} \frac{E_{2}}{\sqrt{\pi}} \frac{E_{1}}{T_{1} \sqrt{\pi}} I_{2,1,1}\left(t_{2}, t_{2}, t_{1}\right)\right],
\end{aligned}
$$

$$
\begin{aligned}
\frac{d C_{2}}{d z}= & -\frac{2 T_{2}}{E_{2}}\left[\frac{C_{2}}{2 T_{2}} \frac{d E_{2}}{d z}+\frac{E_{2} \beta_{22}\left(1+C_{2}^{2}\right)}{2 T_{2}^{3}}+\frac{\gamma E_{2}^{2}}{T_{2}^{2} 2 \sqrt{2 \pi}}\right. \\
& +\frac{\alpha E_{2} C_{2}}{T_{2}^{4} \sqrt{\pi}} I_{2,1,0}\left(t_{2}, t_{2}, 0\right)-\frac{g_{R} E_{2} C_{2}}{T_{2}^{4} \sqrt{\pi}} \frac{E_{1}}{T_{1} \sqrt{\pi}} I_{2,1,1}\left(t_{2}, t_{2}, t_{1}\right) \\
& +\frac{2 \gamma E_{2}}{T_{2}^{2} \sqrt{\pi}}\left(J_{0,1,1}\left(t_{2}, t_{2}, t_{p}\right)+\frac{E_{1}}{T_{1} \sqrt{\pi}} I_{0,1,1}\left(t_{2}, t_{2}, t_{1}\right)\right) \\
& \left.-\frac{4 \gamma E_{2}}{T_{2}^{4} \sqrt{\pi}}\left(J_{2,1,1}\left(t_{2}, t_{2}, t_{p}\right)+\frac{E_{1}}{T_{1} \sqrt{\pi}} I_{2,1,1}\left(t_{2}, t_{2}, t_{1}\right)\right)\right]
\end{aligned}
$$

$$
\begin{aligned}
& \frac{d t_{2}}{d z}=-\frac{1}{E_{2}}\left[\frac{d E_{2}}{d z} t_{2}-E_{2} \beta_{22} \Omega+\frac{\alpha E_{2}}{T_{2} \sqrt{\pi}} I_{1,1,0}\left(t_{2}, t_{2}, 0\right)\right. \\
&\left.-\frac{g_{R} E_{1}}{T_{1} \sqrt{\pi}} I_{1,1,1}\left(t_{1}, t_{2}, t_{1}\right)\right], \\
& \frac{d \Omega_{2}}{d z=}=\frac{1}{E_{2}}\left[\frac{\alpha E_{2} C_{2}}{T_{2}^{3} \sqrt{\pi}} I_{1,1,0}\left(t_{2}, t_{2}, 0\right)-\frac{g_{R} E_{2} C_{2}}{T_{2}^{3} \sqrt{\pi}} \frac{E_{1}}{T_{1} \sqrt{\pi}} I_{1,1,1}\left(t_{2}, t_{1}, t_{2}\right)\right. \\
&-\frac{\alpha E_{2} \Omega_{2}}{T_{2} \sqrt{\pi}} I_{0,1,0}\left(0, t_{2}, 0\right)+\frac{g_{R} E_{2} \Omega_{2}}{T_{2}^{3} \sqrt{\pi}} \frac{E_{1}}{T_{1} \sqrt{\pi}} I_{0,1,1}\left(t_{2}, t_{1}, t_{2}\right) \\
&\left.-\frac{4 \gamma E_{2}}{T_{2}^{3} \sqrt{\pi}}\left(J_{1,1,1}\left(t_{2}, t_{2}, t_{p}\right)+\frac{E_{1}}{T_{1} \sqrt{\pi}} I_{1,1,1}\left(t_{2}, t_{1}, t_{2}\right)\right)\right],
\end{aligned}
$$

where we define the integrals as

$$
\begin{aligned}
& I_{a, b, c}\left(t_{0}, t_{1}, t_{2}\right)=\int_{-\infty}^{\infty}\left(t-t_{0}\right)^{a} f\left(t-t_{1}\right)^{b} f\left(t-t_{2}\right)^{c} \mathrm{~d} t \\
& f\left(t-t_{i}\right)=\exp \left[-\frac{\left(t-t_{i}\right)^{2}}{T_{i}^{2}}\right], \quad \text { where } i=p, 1,2,
\end{aligned}
$$

$$
J_{a, b, c}\left(t_{0}, t_{1}, t_{p}\right)=P_{p 0} I_{a, b, c}\left(t_{0}, t_{1}, t_{p}\right)-P_{h} I_{a, b, c}\left(t_{0}, t_{1}, t_{h}\right) .
$$

Here the subscripts $i=p, 1,2$ correspond to pump, first Stokes, and second Stokes signals, respectively. The relative temporal positions of the pulse centers of the pump and the Stokes signals (the second order Stokes signal is assumed to be the time reference) change mainly due to pulse interactions. Since the frequency shift has a negligible effect, we can ignore the variation in center frequency. However, the temporal shift due to the walk-off effect becomes highly dominant. Thus, we can formulate the change of the pulse temporal positions due to walk off in a reduced form as

$$
\frac{d t_{p}}{d z} \approx w_{p 1}+w_{12}, \quad \frac{d t_{1}}{d z} \approx w_{12}, \quad \text { and } \quad \frac{d t_{2}}{d z} \approx 0
$$

where $w_{p 1}=\int_{\lambda_{p}}^{\lambda_{1}} D(\lambda) \mathrm{d} \lambda \approx \frac{D_{p}+D_{1}}{2}\left(\lambda_{1}-\lambda_{p}\right) \quad w_{12}=\int_{\lambda_{1}}^{\lambda_{2}} D(\lambda) \mathrm{d} \lambda \approx$ $\frac{D_{1}+D_{2}}{2}\left(\lambda_{2}-\lambda_{1}\right)$ stand for the walk-off time between pump-first Stokes $(\sim 0.052 \mathrm{ps} / \mathrm{m})$ and first-second Stokes pulses $(\sim 0.19 \mathrm{ps} / \mathrm{m})$ per unit length and $D$ is the dispersion value (ps/nm-km). 


\section{SIMULATION RESULTS AND STABILITY ANALYSIS}

The main objective of our analysis is to determine if the Stokes pulses converge to DM solitons at the steady state. The split step Fourier method (SSFM) was used to solve the coupled NLS Eqs. (1)-(3). We included GVD, SPM, XPM, and Raman gain mechanisms in our simulations and neglect four-wave mixing (because of the relatively short length of the highly nonlinear DM module) and self-Raman induced frequency shift (because we consider pulses in the ps range) terms. Raman amplification is added as a support interaction. The first and second order Stokes signals are obtained at the output for different pump power and SMF length (determines the net anomalous dispersion) combinations. Figure 3 illustrates the steady-state Stokes pulses inside the cavity (i.e., before the couplers) and their peak power evolution in the laser system as an example of transient behavior toward the steady state. The first Stokes pulse builds up in the system due to proper pumping. If the first Stokes pulse has enough energy (i.e., it reaches threshold for the second Stokes pulse to grow), it starts to generate the second Stokes pulse. While the second Stokes pulse builds up in the system, it depletes the first Stokes. Since the system is highly dissipative, the Stokes pulses reach their steady-state level in a damped oscillatory manner. The magnitude of the overshoot, oscillations, and the settling time in the transient response depend on the pump power level. The temporal deviation of the pulse center of the first Stokes signal results from the temporal walk-off effect.

The envelope of the Stokes pulse is also characterized by comparing it with a Gaussian pulse with the same parameters. The first Stokes pulse is very well represented by a Gaussian profile; however, the tails of the second Stokes pulse are slightly compressed in time so that the Gaussian fitting is less appropriate in this region.

The Stokes pulses repeat themselves after each pass (round trip) at steady state. Thus, the SSFM is employed to propagate the steady-state Stokes pulses for a single pass, so that the intracavity pulse dynamics can be analyzed by extracting the evolution of the pulse parameters. Figure 4 illustrates the evolution of the peak power and the time width, which is defined as the root mean square (RMS) width of the Stokes pulses throughout the cavity at the steady state.

The dynamics of the parameters of each Stokes pulse has a similar behavior. Throughout the DM section of the cavity where all the Raman interactions occur, distributed gain and other nonlinear effects such as SPM and XPM are the dominant terms. Thus, the Stokes pulses are amplified and positively chirped through the DM fibers. Because the dispersion length $\left(L_{D}=T_{0}^{2} /\left|\beta_{2}\right|\right)$ in the DM-HNLF is much larger than the amplification $\left(L_{A}=\left(g_{R}|A|^{2}\right)^{-1}\right)$ and nonlinear lengths $\left(L_{\mathrm{NL}}=\right.$ $\left.\left(\gamma|A|^{2}\right)^{-1}\right)$, and owing to the net zero dispersion of the entire DM map, linear dispersive effects become negligible as seen by the dynamics of the pulse width. However, in the second section of the cavity (standard SMF), since the pulses become separate due to the walk-off, there is virtually no pulse-topulse interaction. Here GVD is the dominant effect throughout the SMF. Since the pulses become highly positively chirped in the DM section of the cavity due to the acquired nonlinear phase, the Stokes pulses first compress and then broaden as seen by the evolutions in Fig. $\underline{4}$ of its peak power and time width.

In order to develop an analytical model to provide a rapid understanding and to explain the pulse dynamics inside the cavity, a detailed variational analysis was employed. The intracavity dynamics of pulse parameters are analyzed in terms of the variational model of Eqs. (13)-(22) as follows. First we use the shooting method in order to satisfy the constraint that the same initial and final values for each pulse parameter are obtained upon a single cavity round trip. Next we explore the intracavity variation of these parameters by solving the same equations for a given steady-state input condition over the entire round trip. To compare the results of the variational analysis with the numerical modeling we investigated the pulse
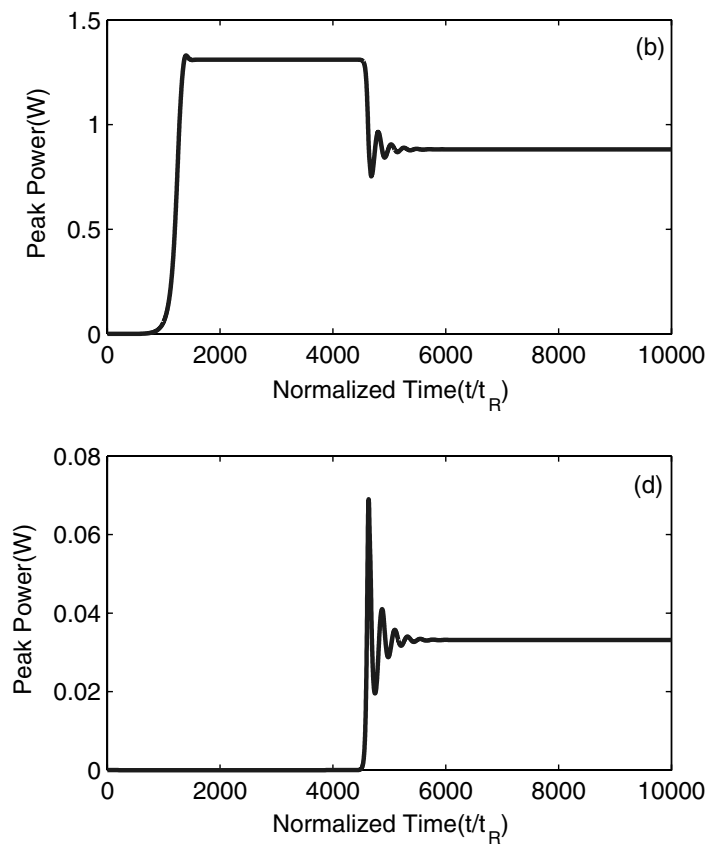

Fig. 3. (a) First order Stokes pulse with (b) Gaussian fitting and the evolution of its peak power and (c) the second order Stokes pulse with (d) Gaussian fitting and the evolution of its peak power at $L_{\mathrm{SMF}}=50 \mathrm{~m}$ and $P_{\text {pump }}=0.72 \mathrm{~W}$. " $t_{R}$ " is the round-trip time of the cavity. 

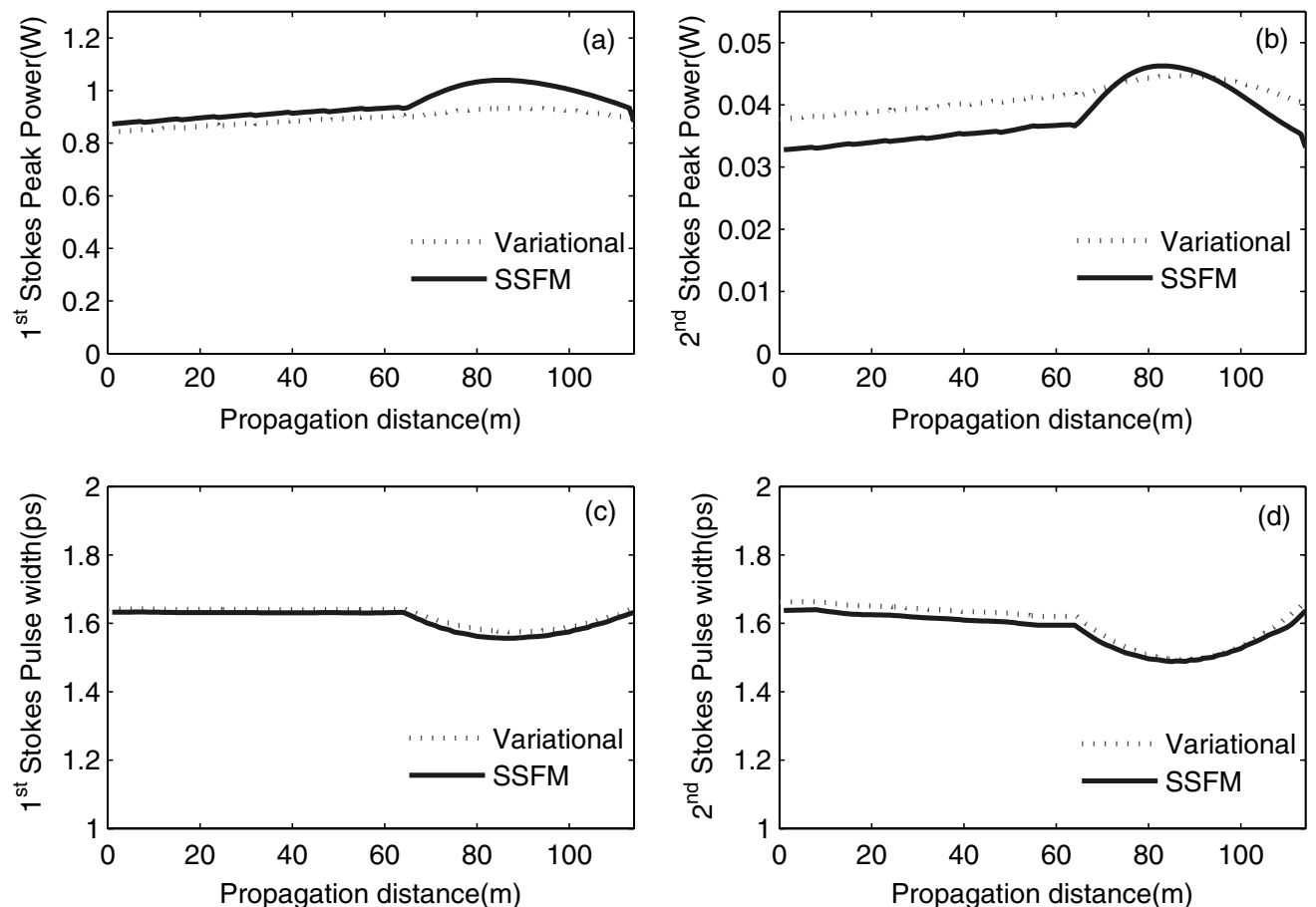

Fig. 4. Intracavity dynamics of the steady-state pulse parameters, peak power, and pulse width of first order Stokes (a-c) and second order Stokes (b-d) at $L_{\mathrm{SMF}}=50 \mathrm{~m}$ and $P_{\text {pump }}=0.72 \mathrm{~W}$. The variational and numerical results are compared.

dynamics for a specific SMF length and pump power $\left(L_{\mathrm{SMF}}=50 \mathrm{~m}\right.$ and $\left.P_{\text {pump }}=0.72 \mathrm{~W}\right)$ as shown again in Fig. $\underline{4}$. The variational equations are quite successful at explaining the general trend of the pulse parameters at the steady state. Since the variational analysis assumes that the pulse shape does not change throughout the propagation, all perturbations such as Raman gain, pump depletion, and the nonlinear chirp (induced by XPM) affect the entire pulse by changing its total energy and phase. However, due to temporal walk off and the mismatches between the various pulse widths, these

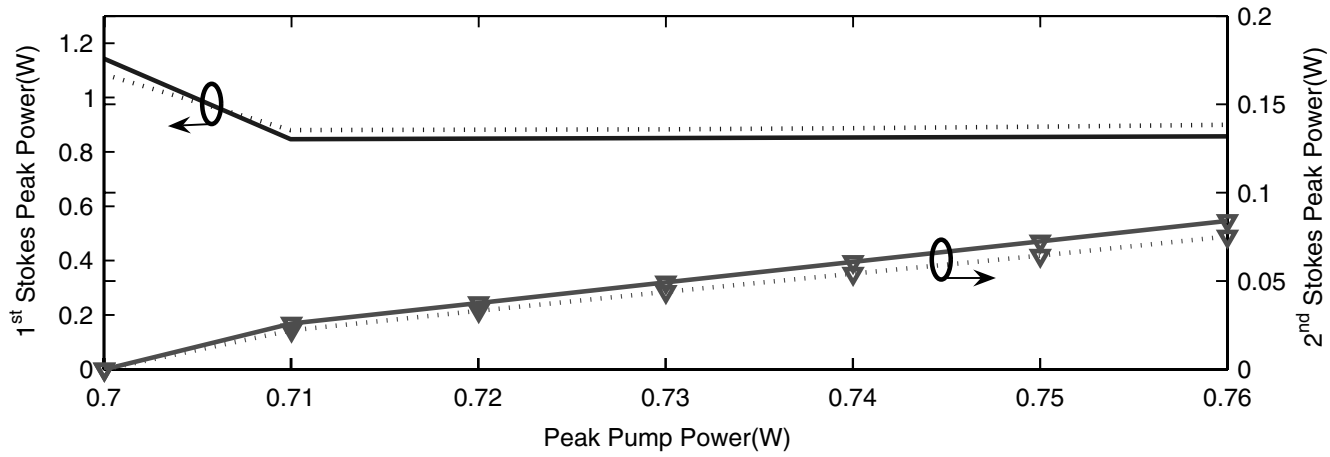

(a)

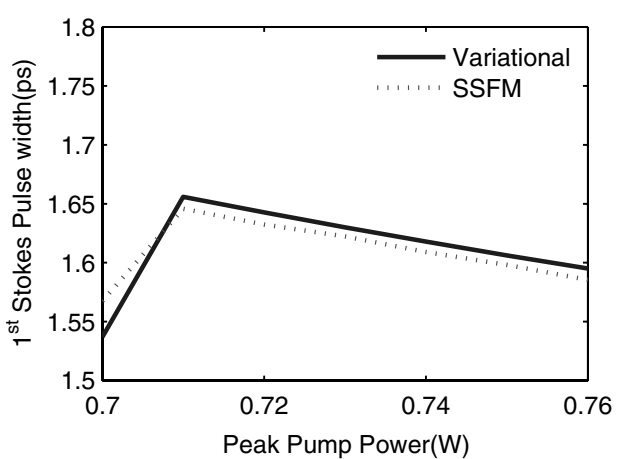

(b)

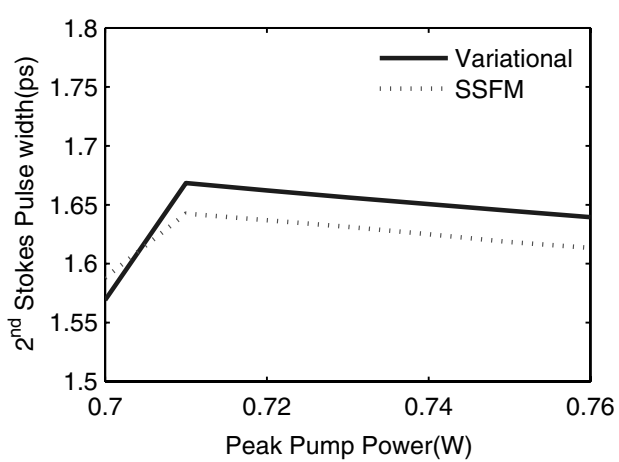

(c)

Fig. 5. Steady-state peak power (a) and pulse width (b-c) of the Stokes pulses with respect to peak pump power at $L_{\mathrm{SMF}}=50 \mathrm{~m}$. Analytical (solid) and numerical results (dotted) are also illustrated as a comparison. 


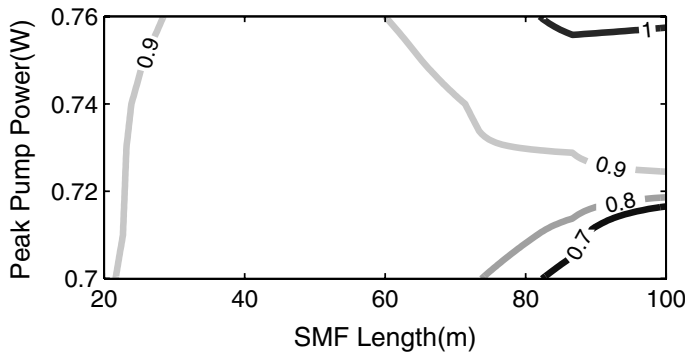

(a)

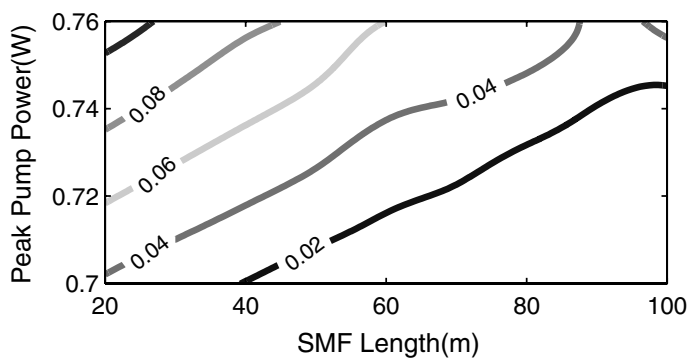

(c)

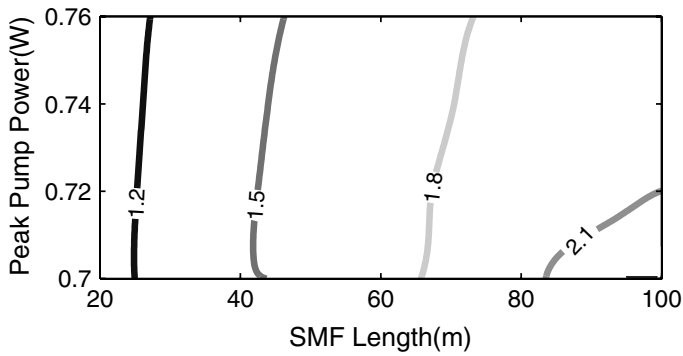

(b)

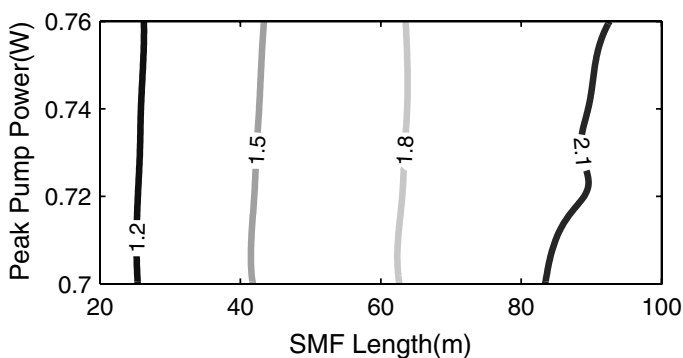

(d)

Fig. 6. Contour plots for the peak power (W) and pulse width (ps) of the first order Stokes pulse (a-b) and the contour plots for the peak power (W) and pulse width (ps) of the second order Stokes pulse (c-d) with respect to the SMF length and peak pump power.

perturbations force the pulses to have local changes at different positions. Thus the variational analysis is accompanied by an intrinsic averaging mechanism that reduces the effect of the gain and the chirp (arising mainly from XPM) over the central region of each pulse, which explains the small deviation between variational and numerical results.

In addition to the intracavity pulse dynamics, steady-state pulse parameters such as the peak power and the time width (RMS width) of the first and the second order Stokes inside the cavity (i.e., before the couplers) that are obtained for different peak pump power levels are extracted by the variational analysis and illustrated in Fig. 5, along with the corresponding numerical results. The overall agreement with the numerical results obtained by the SSFM confirms the validity of our variational analysis.

We also investigate the stable pulse generation regime, which is defined by the boundaries of either the Stokes threshold on the one hand and the nonlinear induced pulse breakup on the other hand, for various SMF lengths (net dispersion) ranging from $20 \mathrm{~m}$ to $100 \mathrm{~m}$ and for peak pump powers from $0.7 \mathrm{~W}$ to $0.76 \mathrm{~W}$. Clearly the stability of our pulse solutions in this regime is due to the attractive nature of the strongly dissipative laser map under consideration. The results are reported in the contour plots of Fig. $\underline{6}$, which show the peak power and time width (RMS width) of first and second order Stokes pulses inside the cavity (i.e., before the couplers) that converge at steady state. Figure 6 shows that stable first order Stokes pulses with up to $1 \mathrm{~W}$ peak power and $1 \mathrm{ps}$ to $2.1 \mathrm{ps}$ pulse widths are generated. Similarly, second order Stokes pulses with $0.02 \mathrm{~W}$ to $0.1 \mathrm{~W}$ peak power and $1 \mathrm{ps}$ to $2.1 \mathrm{ps}$ pulse widths can be obtained. At low pump powers that are very close to threshold only, the first order Stokes can be generated. When the pump power is increased, the first order Stokes signal starts to build up in the system and converge to a steady state. If the final peak power of the first Stokes signal reaches the threshold for second Stokes signal, it generates the second Stokes pulse. At high pump powers, due to imbalance between strong nonlinearity and weak dispersion, the first Stokes signal starts to break up and the laser system becomes unstable. The robustness to nonlinearity increases proportionally to the net dispersion (SMF length).

\section{CONCLUSION}

We investigated the stability of DM synchronous Raman lasers up to second order both analytically and numerically. The variational analysis was conducted in order to derive the differential equations that explain the intracavity dynamics of Stokes pulses in terms of their pulse parameters. The resulting coupled differential equations with periodic boundary conditions are solved to obtain the Stokes pulse parameters at the steady state. The analytically and numerically derived pulse parameters of the Stokes pulses are compared to illustrate the correctness of the analytical predictions. The results show that stable second order Raman Stokes pulses with $0.02 \mathrm{~W}$ to $0.1 \mathrm{~W}$ peak power and $1 \mathrm{ps}$ to $2.1 \mathrm{ps}$ pulse widths can be achieved in a DM Raman laser system. Results can be further improved by optimizing the parameters of the dispersion management.

\section{ACKNOWLEDGMENTS}

This work is supported by the DARPA Remoted Analog-toDigital Converter with De-serialization and Reconstruction (RADER) program

\section{REFERENCES}

1. A. A. M. Staring, L. H. Spiekman, J. J. M. Binsma, E. J. Jansen, T. V. Dongen, P. J. A. Thijs, M. K. Smit, and B. H. Verbeek, "A compact nine-channel multiwavelength laser," IEEE Photon. Technol. Lett. 8, 1139-1141 (1996).

2. M. Zirngibl, C. H. Joyner, C. R. Doerr, L. W. Stulz, and H. M. Presby, "An 18-channel multifrequency laser," IEEE Photon. Technol. Lett. 8, 870-872 (1996).

3. M. D. Mermelstein, C. Horn, S. Radic, and C. Headley, "Sixwavelength Raman fibre laser for $C$ - and $L$-band Raman 
amplification and dynamic gain flattening," Electron. Lett. 38, 636-638 (2002).

4. C. J. S. Matos, S. V. Popov, and J. R. Taylor, "Short-pulse, all-fiber, Raman laser with dispersion compensation in a holey fiber," Opt. Lett. 28, 1891-1893 (2003).

5. C. J. S. De Matos, D. A. Chestnut, P. C. Reeves-Hall, F. Koch, and J. R. Taylor, "Multi-wavelength, continuous wave fiber Raman ring laser operating at $1.55 \mu \mathrm{m}$," Electron. Lett. 37, 825-826 (2001).

6. X. Y. Dong, P. Shum, N. Q. Ngo, and C. C. Chan, "Multiwavelength Raman fiber laser with a continuously tunable spacing," Opt. Express 14, 3288-3293 (2006).

7. J. H. V. Price, K. Furusawa, T. M. Monro, L. Lefort, and D. J. Richardson, "Tunable, femtosecond pulse source operating in the range $1.06-1.33 \mu \mathrm{m}$ based on an $\mathrm{Yb}^{3+}$-doped holey fiber amplifier," J. Opt. Soc. Am. B 19, 1286-1294 (2002).

8. M. D. Mermelstein, C. Headley, J.-C. Bouteiller, P. Steinvurzel, C. Horn, K. Feder, and B. J. Eggleton, "A high-efficiency power-stable three-wavelength configurable Raman fiber laser," in Proceedings of the Optical Fiber Communication Conference, 2001 OSA Technical Digest Series (Optical Society of America, 2001), paper PD3.

9. M. D. Mermelrtein, C. Horn, Z. Huang, M. Luvalle, 1. X. Routeiller, C. Headley, B. J. Eggleton, "Configurability of a three-wavelength Raman fiber laser for gain ripple minimization and power partitioning," in Proceedings of the Optical Fiber Communication Conference, A. Sawchuk, ed., Vol. 70 of OSA Trends in Optics and Photonics (Optical Society of America, 2002), paper TuJ2.

10. C. Headley, "Configurable multiple-wavelength all-fiber laser for efficient stable Raman amplification," in Proceedings of the Optical Fiber Communication Conference (OFC), A. Sawchuk, ed., Vol. 70 of OSA Trends in Optics and Photonics (Optical Society of America, 2002), paper TuB1.
11. X. Normandin, F. Leplingard, E. Bourova, C. Ledere, T. Lopez, L. J. Guerin, and D. Bayart, "Experimental assessment of phospho-silicate fibers for three-wavelength $(1427 \mathrm{~nm}, 1455 \mathrm{~nm}$, $1480 \mathrm{~nm}$ ) reconfigurable Raman lasers," in Proceedings of the Optical

Fiber Communication Conference, A. Sawchuk, ed., Vol. 70 of OSA Trends in Optics and Photonics (Optical Society of America, 2002), paper TuB2.

12. M. Müller and J. M. Schins, "Imaging the thermodynamic state of lipid membranes with multiplex CARS microscopy," J. Phys. Chem. B 106, 3715-3723 (2002).

13. J. X. Cheng, A. Volkmer, L. D. Book, and X. S. Xie, "Multiplex coherent anti-Stokes Raman scattering micro spectroscopy and study of lipid vesicles," J. Phys. Chem. B 106, 8493-8498 (2002).

14. T. W. Kee and M. T. Cicerone, "Simple approach to one-laser, broadband coherent anti-Stokes Raman scattering microscopy," Opt. Lett. 29, 2701-2703 (2004).

15. H. A. Haus and M. Nakazawa, "Theory of the fiber Raman soliton laser," J. Opt. Soc. Am. B 4, 652-660 (1987).

16. S. K. Kalyoncu, S. Gao, E. K. Tien, Y. Huang, D. Yildirim, E. Adas, S. Wabnitz, and O. Boyraz, "Stability analysis of second order pulsed Raman laser in dispersion managed systems," Proc. SPIE 8071, 807116(2011).

17. G. P. Agrawal, Nonlinear Fiber Optics, 4th ed. (Academic, 2009).

18. C. Antonelli, J. Chen, and F. X. Kartner, "Intracavity pulse dynamics and stability for passively mode-locked lasers," Opt. Express 15, 5919-5924 (2007).

19. A. Hook, D. Anderson, and M. Lisak, "Effects of cross-phase modulation and pump depletion on Stokes pulse dynamics in stimulated Raman scattering,” J. Opt. Soc. Am. B 6, 1851-1858 (1989).

20. OFS Specialty Photonics Division, http://ofscatalog specialtyphotonics.com. 